in appearance and flavor was the extrudate with $3.5 \%$ of seaweed. The antioxidant capacity and ACE inhibitory effect persisted after in vitro gastrointestinal digestion and was higher for the dialysate with seaweed due to phenolic compounds and bioactive peptides generated by hydrolysis from the seaweed, which would be potentially bio-accessible.

\title{
Biología de abejas silvestres potencialmente utilizables como polinizadores de cultivos
}

\section{Milagros Dalmazzo}

milidalmazzo@yahoo.com

Dr. Arturo Roig-Alsina

División Entomología, Museo Argentino de Ciencias Naturales "Bernardino Rivadavia". Av. Ángel Gallardo 470, Ciudad Autónoma de Buenos Aires

Cátedra Entomología, Departamento de Ciencias Naturales, Facultad de Humanidades y Ciencias

Universidad Nacional del Litoral

Fecha de la defensa: 20/03/2013

\section{Resumen}

Las abejas (Apoidea, Hymenoptera) son el grupo de insectos que por su diversidad, alimentación y comportamiento en general, ejercen la acción polinizadora de mayor importancia. La especie más utilizada en polinización es la abeja doméstica, Apis mellifera L., de todos modos hay estudios que han demostrado que en muchos cultivos las abejas silvestres son más eficientes.

Debido a la carencia de conocimiento de la biología de las especies de abejas nativas de nuestro país, y de la región, y a que el empleo de polinizadores en cultivos comerciales está en auge, aparece como alternativa la importación de especies polinizadoras exóticas para su uso en cultivos comerciales. Sin embargo está comprobado el efecto negativo de estas introducciones.

En Argentina fue importada en forma sostenida la especie Megachile rotundata para producción de semilla de alfalfa. En 2011 los organismos nacionales de control, SENASA y Dirección Nacional de Fauna Silvestre, suspendieron la importación de esta megachila y desaprobaron los intentos de importación de otras especies. Por tal motivo se hace perentorio el conocimiento de las especies nativas que cumplan la función polinizadora, para lo cual se debe comenzar a estudiar las mismas desde el punto de vista sistemático y biológico, de modo que en una etapa posterior, se pueda evaluar su adaptabilidad a la cría comercial en consonancia con prácticas de manejo ecológicamente sustentables.

En la presente tesis, se han elegido especies del género Augochlora (Halictidae, Apoidea) que nidifican en sustrato altamente transportable y podrían presentar algún grado de socialidad y hábitos alimentarios no especializados, cualidades que le otorgan alta factibilidad de manejo. De esta manera se propuso estudiar aspectos sistemáticos y biológicos desconocidos para estas especies, con especial interés en aquellos comportamientos que permitan su posterior utilización en polinización.

Se realizó una revisión sistemática de las 
especies del género Augochlora presentes en la región templada de Argentina y Uruguay. Se seleccionaron las especies Augochlora amphitrite y Augochlora phoemonoe para el estudio de aspectos biológicos relacionados a la nidificación, estructura social, fenología y desarrollo. Estos parámetros fueron estudiados en el campo a través de observaciones de nidos en condiciones naturales y en laboratorio, a través de observaciones en nidos artificiales mantenidos en un insectario. Se estudió el hábito alimentario mediante análisis polínico de reservas de nidos hallados en el campo y observaciones de visitas florales.

Se encontraron cinco especies en el área central de Argentina y Uruguay, región de gran importancia agrícola y forrajera delimitada por el paralelo $30^{\circ}$ y $40^{\circ}$ latitud sur: A. iphigenia (Holmberg), A. amphitrite (Schrottky), A. nausicaa (Schrottky), A. phoemonoe (Schrottky) y A. daphnis Smith. Las cuatro primeras son elementos comunes de la fauna de abejas de Argentina, la quinta especie, de Montevideo, Uruguay, es conocida solo por el ejemplar tipo. Se presentan re-descripciones de todas las especies, ilustraciones, datos de distribución y una clave taxonómica. Se designa el lectotipo de Augochlora iphigenia Holmberg, 1886. Oxystoglossa semiramis Schrottky, 1911 y Halictus brochidens Vachal, 1911 son nuevos sinónimos de A. iphigenia Holmberg. Odontochlora thebe Schrottky, 1909 es un nuevo sinónimo de Odontochlora amphitrite Schrottky, 1909. Se presenta una lista de especies de Augochlora presentes en Argentina, de las cuales ocho son nuevas citas para el país. Se pudo establecer que la diversidad y composición de especies varía según la región biogeográfica y que de acuerdo con la diversidad obser- vada en países vecinos como Brasil, Paraguay y Bolivia, las regiones Paranaense y de Yungas podrían contener muchas más especies que las registradas en las colecciones entomológicas examinadas. A su vez la composición de especies sería diferente entre estas regiones. La diversidad decrece abruptamente al ingresar a la región templada Pampeana donde es posible encontrar solo cinco especies. El género está ausente en las áreas xéricas como las regiones del Monte, Patagonia y Andinas en Argentina, como así también en Chile.

Se describe la estructura de nidificación de $A$. amphitrite y $A$. phoemonoe. Ambas especies presentaron dos tipos de estructura de nidificación, racimos de celdas sostenidos por pilares a una cavidad y celdas apoyadas al sustrato sin pilares. Esta variabilidad, no exhibida por otros Augochlorinos que nidifican en el suelo, estaría relacionada a la dureza del sustrato de nidificación.

En $A$. amphitrite y $A$. phoemonoe fue observada la convivencia y cooperación entre dos o más hembras adultas dentro de un mismo nido. En el caso de las observaciones de insectario, este grupo de hembras estaba conformado por la madre, hembra totipotente fundadora del nido y las hijas. Se observaron interacciones entre este grupo de hembras y división de tareas indicando la existencia de un grado de socialidad verdadera. El análisis de las frecuencias de actividades e interacciones realizadas por cada individuo dentro del insectario permitió encontrar diferencias estadísticamente significativas entre hembras fundadoras y hembras hijas que posibilitaron describir al menos dos castas: reinas reproductoras y obreras.

En relación a la alimentación se encontró que $A$. amphitrite y $A$. phoemonoe son espe- 
cies polilécticas colectando polen de diez y tres familias vegetales. Los registros de visitas florales permitieron ampliar el espectro floral de estas dos especies para las cuales eran desconocidos sus hábitos de forrajeo.

La información obtenida constituye uno de los primeros aportes al estudio de la biología del subgénero Augochlora y permitió descubrir el comportamiento eusocial en un subgénero que, hasta la presente tesis, era considerado de especies solitarias. Se ha realizado un estudio detallado del comportamiento social e interacciones de dos especies de Augochlora. Se ha realizado un aporte de interés para el estudio de la variación y evolución de la socialidad en Augochlorini. Estos aportes invitan a reconsiderar la rigidez de las categorías clasificatorias utilizadas en Augochlorinos.

Se pudo obtener la cría artificial de las dos especies en condiciones de laboratorio y delinear las estrategias a tener en cuenta para la cría comercial de estas especies con el objetivo de manejarlas como polinizadoras de cultivos hortícolas. La presente tesis constituye un aporte novedoso al estudio de dos especies de abejas silvestres potencialmente utilizables como polinizadoras de cultivos en la región central de la Argentina.

Biology of wild bees as prospective crop pollinators

\section{Summary}

Use and management of wild bees in crop pollination are growing up around the world. Despite this, studies on the biology of wild bees as pollinators are almost nonexistent in Argentina. The purpose of the present thesis is to study the systematics and biological aspects of Augochlora species. I chose Augochlora amphitrite and Augochlora phoemonoe for the biological study. Both species had interesting characteristics for their management. Only five species occur in central Argentina and Uruguay, between $30^{\circ}$ and $40^{\circ}$ south latitude: $A$. iphigenia Holmberg, A. amphitrite (Schrottky), A. nausicaa (Schrottky), A. phoemonoe (Schrottky) and $A$. daphnis Smith. Re-descriptions of all species, illustrations, distributional data, and taxonomic keys are presented.

The nest structure of $A$. amphitrite and A. phoemonoe are described. Two types of nest architecture were found in both species. The nest architecture differed according to the substrate where they were built. Coexistence and cooperation of two or three adult females into the same nest were observed in A. amphitrite and $A$. phoemonoe. In the bee room, these females were the mother, a totipotent foundress, and its daughters. Interactions and division of labour between these females were observed, showing that both studied species were primitively eusocial. The analysis of frequency of activities and interactions recorded for each individual into the bee room, gave statistically significant differences between the foundress female and the daughters.

Augochlora amphitrite and A. phoemonoe used pollen from ten and three families of plants respectively. For this reason, these bee species can be classified as polylectic. A detailed study of the behavioral interactions and social structure of two species of Augochlora was performed. This study contributes to the interpretation of the variation and evolutionary mechanisms of eusociality in Augochlorini. 